\title{
Comparative Evaluation of Intron Prediction Methods and Detection of Plant Genome Annotation Using Intron Length Distributions
}

\author{
Long Yang ${ }^{1}$ and Hwan-Gue $\mathrm{Cho}^{2 *}$ \\ ${ }^{1}$ Tobacco Laboratory, Shandong Agricultural University, \\ Shandong 271-018, China, ${ }^{2}$ Graphics Application \\ Laboratory, Department of Computer Science and \\ Engineering, Pusan National University, Busan 609-735, \\ Korea
}

\begin{abstract}
Intron prediction is an important problem of the constantly updated genome annotation. Using two model plant (rice and Arabidopsis) genomes, we compared two well-known intron prediction tools: the Blast-Like Alignment Tool (BLAT) and Sim4cc. The results showed that each of the tools had its own advantages and disadvantages. BLAT predicted more than $99 \%$ introns of whole genomic introns with a small number of false-positive introns. Sim4cc was successful at finding the correct introns with a false-negative rate of $1.02 \%$ to $4.85 \%$, and it needed a longer run time than BLAT. Further, we evaluated the intron information of 10 complete plant genomes. As non-coding sequences, intron lengths are not limited by a triplet codon frame; so, intron lengths have three phases: a multiple of three bases (3n), a multiple of three bases plus one $(3 n+1)$, and a multiple of three bases plus two $(3 n+2)$. It was widely accepted that the percentages of the $3 n, 3 n+1$, and $3 n+2$ introns were quite similar in genomes. Our studies showed that $80 \%(8 / 10)$ of species were similar in terms of the number of three phases. The percentages of $3 n$ introns in Ostreococcus lucimarinus was excessive (47.7\%), while in Ostreococcus tauri, it was deficient (29.1\%). This discrepancy could have been the result of errors in intron prediction. It is suggested that a three-phase evaluation is a fast and effective method of detecting intron annotation problems.
\end{abstract}

Keywords: intron length distributions, intron prediction, plant, three phases

*Corresponding author: E-mail: hgcho@pusan.ac.kr Tel +82-51-510-2871, Fax +82-51-582-5009

Received 2 February 2012, Revised 15 February 2012, Accepted 17 February 2012

\section{Introduction}

With more and more species' genomes completely sequenced, noncoding sequences have become a focus of researchers' attention, especially for the study of introns. In order to facilitate further research, a number of intron databases have been developed (Table 1). The number of plant intron databases is much smaller than that in mammals and only in several model plants (such as Arabidopsis and rice). Using known genome sequences and coding sequences (expressed sequence tags [ESTs] or cDNA), introns can be detected by aligning coding sequences with genome sequences. Many tools were developed to detect introns in eukaryotes (Table 2) [1-16]. These tools used different algorithms and computer languages (such as Java, $\mathrm{C}++$, and Python) to predict introns.

Therefore, the question is: there are many intron databases, algorithms, and detection methods for the study of eukaryotes, but which among them are the most suitable for the detection of plant introns? Among these tools, the Blast-Like Alignment Tool (BLAT) and Sim4cc are the most commonly used tools. BLAT applies in genomewide alignment [11]. Sim4cc is a tool for aligning cDNA and genomic sequences between species at various evolutionary distances [2]. Rice and Arabidopsis, as monocotyledonous and dicotyledonous model plants, are widespread with regard to in-depth research. Their genome sequences have been annotated in detail, including their gene sequences, complementary DNA (cDNA) sequences, coding DNA sequence (CDS) sequences, exon sequences, intron sequences, and intergene sequences. Therefore, it is possible to use this model plant information to test these intron prediction tools.

Genome annotation is a difficult and accurate projecteven the best-annotated or most carefully studied genomes are continually re-released; e.g., release 7 of the Rice Genome Annotation Project was available on October 31, 2011 (http://rice.plantbiology.msu.edu/). But, determining the accuracy and detecting the inherent errors of the genome annotation is a problem. Since introns are removed from protein-coding transcripts, intron lengths are not expected to respect coding frames across the genome [17]. Using intron length distributions, Roy and Penny [18] point out a rapid and simple method for de- 


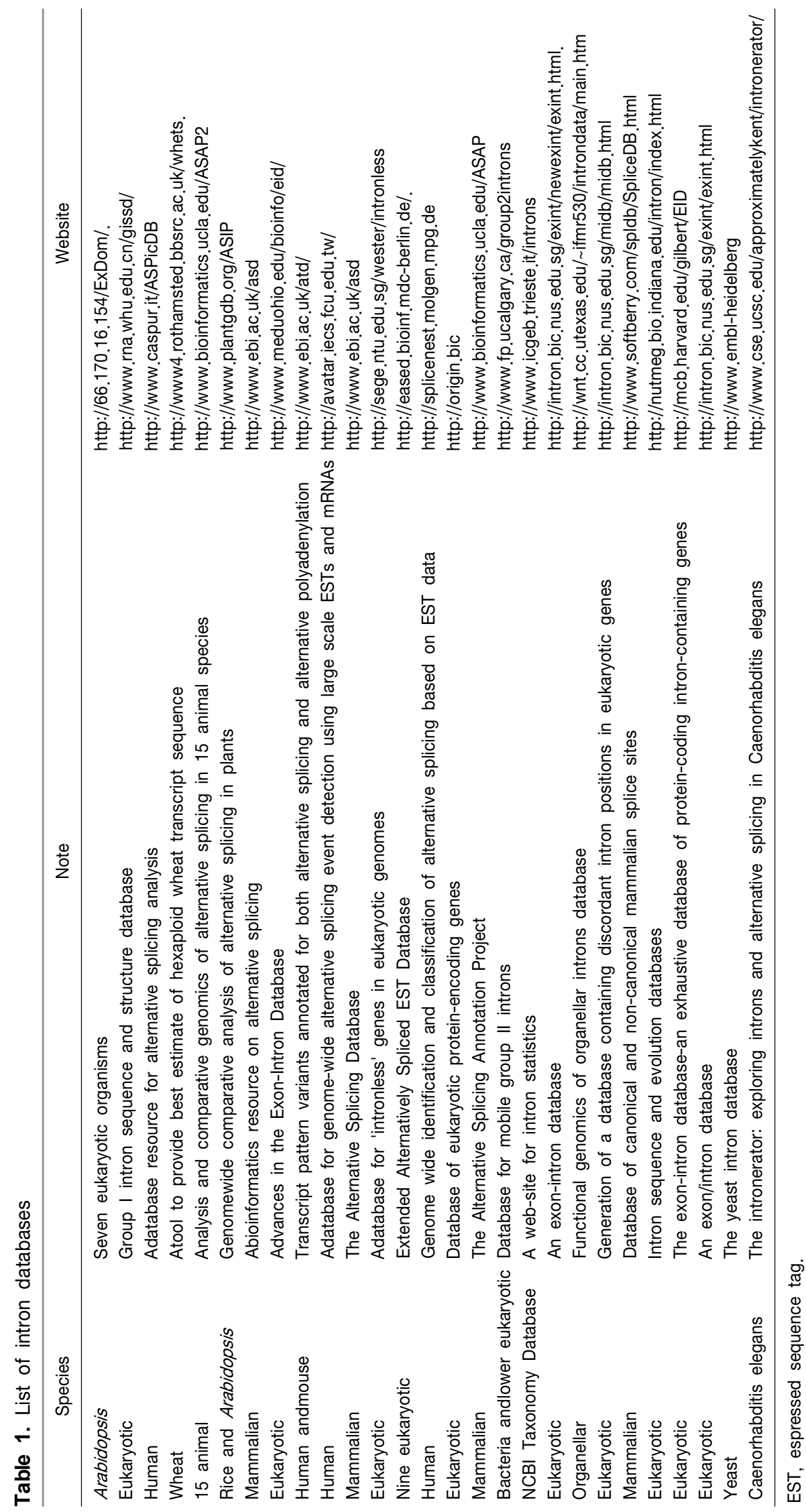


60 Genomics \& Informatics Vol. 10(1) 58-64, March 2012

Table 2. Tools for detection alternative-splicing/introns

\begin{tabular}{|c|c|c|}
\hline Tools name & Description & Reference \\
\hline FIRMA & A method for detection of alternative splicing from exon array data & Purdom et al. [1] \\
\hline $\operatorname{Sim} 4 \mathrm{cc}$ & A cross-species spliced alignment program & Zhou et al. [2] \\
\hline Sircah & A tool for the detection and visualization of alternative transcripts & Harrington and Bork [3] \\
\hline Splicy & $\begin{array}{l}\text { A web-based tool for the prediction of possible alternative splicing events from Affymetrix } \\
\text { probeset data }\end{array}$ & Rambaldi et al. [4] \\
\hline WhETS & A tool to provide best estimate of hexaploid wheat transcript sequence & Mitchell et al. [5] \\
\hline RRE & $\begin{array}{l}\text { A tool for the extraction of non-coding regions surrounding annotated genes from genomic } \\
\text { datasets }\end{array}$ & Lazzarato et al. [6] \\
\hline ESTMAP & A system for expressed sequence tags mapping on genomic sequences & Milanesi and Rogozin [7] \\
\hline MapSplice & Accurate mapping of RNA-seq reads for splice junction discovery & Wang et al. [8] \\
\hline HMMSplicer & $\begin{array}{l}\text { A tool for efficient and sensitive discovery of known and novel splice junctions } \\
\text { in RNA-Seq data }\end{array}$ & Dimon et al. [9] \\
\hline EUGÈNE'HOM & A generic similarity-based gene finder using multiple homologous sequences & Foissac et al. [10] \\
\hline BLAT & The BLAST-like alignment tool & Kent [11] \\
\hline ASAP & $\begin{array}{l}\text { A novel method to predict the exon-intron structure of a gene that is optimally compatible } \\
\text { to a set of transcript sequences }\end{array}$ & Lee et al. [12] \\
\hline EVOPRINTER & A multigenomic comparative tool for rapid identification of functionally important DNA & Odenwald et al. [13] \\
\hline GenoMiner & A tool for genome-wide search of coding and non-coding conserved sequence tags & Castrignanò et al. [14] \\
\hline Restauro-G & A rapid genome re-annotation system for comparative genomics & Tamaki et al. [15] \\
\hline Scan Intron & Scan a database of introns confirmed by cDNA/EST alignments for patterns at either end & Kent and Zahler [16] \\
\hline
\end{tabular}

BLAT, Blast-Like Alignment Tool.

Table 3. Ten plant species genome sequence sources

\begin{tabular}{llll}
\hline \multicolumn{1}{c}{ Species } & \multicolumn{1}{c}{ Version } & \multicolumn{1}{c}{ Source } & \multicolumn{1}{c}{ Reference } \\
\hline Arabidopsis thaliana & TAIR, version 10 & http://www.arabidopsis.org/ & Swarbreck et al. [19] \\
Oryza sativa L. ssp. japonica & Release 7 & http://rice.plantbiology.msu.edu/ & Goff et al. [20] \\
Oryza sativa L. ssp. indica & 28 Oct, 2008 & http://rice.genomics.org.cn/ & Yu et al. [21] \\
Zea mays & B73_RefGen_v2 & http://www.maizegdb.org/ & Schnable et al. [22] \\
Sorghum bicolor & Version 1.0 & http://www.phytozome.net/sorghum.php & Paterson et al. [23] \\
Cucumis sativus & 7 April, 2011 & http://cucumber.genomics.org.cn/ & Han et al. [24] \\
Chlamydomonas reinhardtii & Version 4.0 & http://genome.jgi-psf.org/Chlre4/ & Merchant et al. [25] \\
Ostreococcus lucimarinus & Version 2.0 & http://genome.jgi-psf.org/Ost9901_3/ & Palenik et al. [26] \\
Ostreococcus tauri & Version 2.0 & http://genome.jgi-psf.org/Ostta4 & Palenik et al. [26] \\
Medicago truncatula & Mt3.5.1 & http://www.medicago.org// & Young et al. [27] \\
\hline
\end{tabular}

tecting a variety of possible systematic biases in gene prediction or even problems with genome assemblies. Roy's method showed that a good genome annotation is accepted as roughly equal proportions of intron lengths of three phases: a multiple of three bases (3n), one more than a multiple of three bases $(3 n+1)$, and two more $(3 n+2)$. Skewed predicted intron length distributions thus suggest systematic errors in intron prediction. But, many plants with sequenced genomes have not been commented on.

In this study, we compared the advantages and disadvantages of BLAT and Sim4cc for model plants' intron predictions, and we attempted to find a better way to predict the intron information of plants. Based on Roy's method, we evaluated the intron information of 10 plant genomes and discuss a skew in genome wide in- tron length distributions that indicates systematic problems with intron predictions.

\section{Methods}

\section{Genome sequences}

Ten plant genome sequences and transcript (EST, CDS, or CDNA) sequences were downloaded and indicated in Table 3 [19-27]. Table 3 contains the name of the 10 plant species, source websites, and genome sequence versions used in this study. 


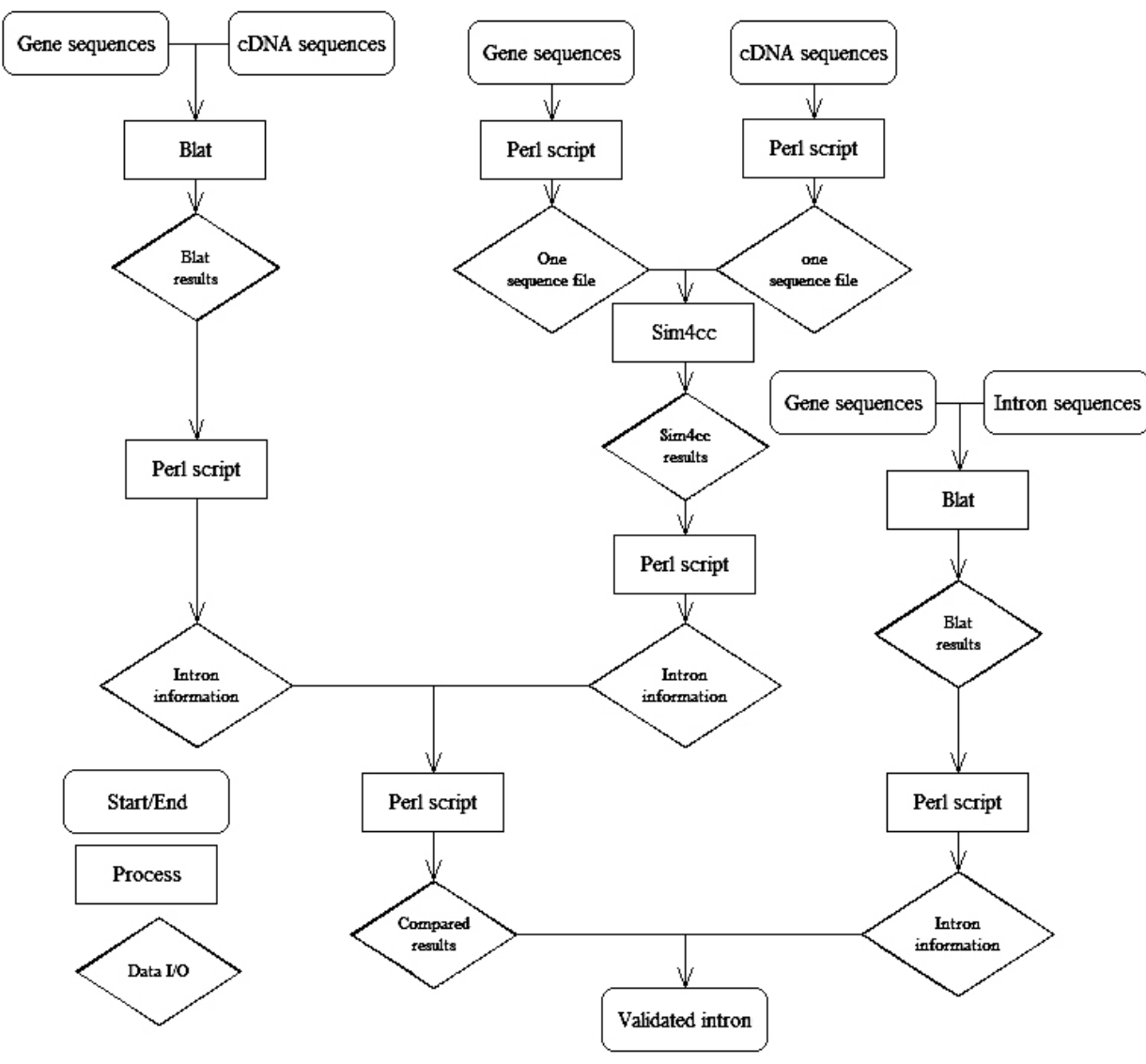

Fig. 1. Flowchart of a comparison of BLAT and Sim4cC results in predicting introns. Intron information, including the following information of one intron: gene name, intron number, intron position in the gene, intron length, intron position in the genome, forward-exon length, backward-exon length, and intron sequences. BLAT, BlastLike Alignment Tool.

\section{Comparative BLAT and Sim4cc analysis}

Using cDNA sequences and gene sequences, we searched rice and Arabidopsis introns by two methods -BLAT and Sim4cc- and then compared the results with annotated information.

The steps of this method are as follows (Fig. 1): 1) Using the gene sequences of BLAT with its own cDNA sequences, we found intron information from the BLAT results by Perl script. 2) We sliced gene sequences and cDNA sequences to folders by Perl script. In these folders, there was one sequence per file, and the gene name was the file name. Using the same gene name of the gene and cDNA file, we blasted the gene sequences and cDNA sequences using Sim4cc. Then, we got intron information from the Sim4cc results by Perl script. 3) We compared the results of the two types of software (BLAT and Sim4cc) and then got the annotated intron information. 4) We aligned intron sequences with their own gene sequences to develop detailed intron information, such as the intron position in the gene, intron length, intron number, forward-exon length, and backward-exon length, etc. 5) We compared the results from the two types of software with the annotated information to validate the methods.

\section{Intron length distributions analysis}

Using Perl script, we extracted the intron information of the 10 plant genomes from the genome annotation. Then, we counted the number and percentage of $3 n, 3 n$ +1 , and $3 n+2$ of these 10 plants' intron length distributions.

\section{Results and Discussion}

\section{A comparison of BLAT and Sim4cc}

As a prerequisite, it was assumed that the intron annotated information was correct and complete. Then, the software's results were compared with the annotated information. Three sets of results of intron information were obtained: two sets from the software (BLAT and Sim4cc) and one set from the annotated information (Table 4).

Using BLAT, we found $99.35 \%$ and $99.87 \%$ of the introns of all rice and Arabidopsis annotated introns, respectively. These introns were almost all of the introns in the genome - that is, only $0.13 \%$ to $0.65 \%$ of the introns were not found. In contrast, by using Sim4cc, $95.15 \%$ to $98.98 \%$ of the introns were found $(1.02 \%$ to 
62 Genomics \& Informatics Vol. 10(1) 58-64, March 2012

Table 4. Compared BLAT and Sim4cc predicted intron information with annotated intron information

\begin{tabular}{|c|c|c|c|c|c|c|c|c|c|c|c|c|}
\hline \multirow{3}{*}{ Species } & \multicolumn{4}{|c|}{ Annotated } & \multicolumn{4}{|c|}{ BLAT } & \multicolumn{4}{|c|}{$\operatorname{Sim} 4 c c$} \\
\hline & \multirow{2}{*}{ Intron No. } & \multirow{2}{*}{ Gene No. } & \multicolumn{2}{|c|}{ Gene (with intron) } & \multicolumn{2}{|c|}{ Intron } & \multicolumn{2}{|c|}{ Gene (with intron) } & \multicolumn{2}{|c|}{ Intron } & \multicolumn{2}{|c|}{ Gene (with intron) } \\
\hline & & & No. & $\%$ & No. & $\%$ & No. & $\%$ & No. & $\%$ & No. & $\%$ \\
\hline Rice & 251,812 & 56,797 & 44,796 & 78.87 & 250,178 & 99.35 & 44,370 & 78.12 & 239,590 & 95.15 & 42,577 & 74.96 \\
\hline Arabidopsis & 175,513 & 41,671 & 30,177 & 72.42 & 175,285 & 99.87 & 30,194 & 72.46 & 173,715 & 98.98 & 29,875 & 71.69 \\
\hline
\end{tabular}

BLAT, Blast-Like Alignment Tool.

Table 5. Comparative comparison of BLAT and Sim4cc in intron prediction

\begin{tabular}{lccccc}
\hline Tools & False-positive (\%) & False-negative $(\%)$ & Accuracy $(\%)$ & Operability & Running time \\
\hline BLAT & 0.38 & 0.39 & 99.62 & Easy & Fast \\
Sim4cc & 0 & 2.94 & 100 & Complex & Slow \\
\hline
\end{tabular}

Note: In this table, the data is the average of two model plants (Arabidopsis and rice).

BLAT, Blast-Like Alignment Tool.

$4.85 \%$ of the introns were lost) of all rice and Arabidopsis annotated introns. In summary, BLAT got more of the introns in a genome than Sim4cc. In light of this result, it seems as though that BLAT produces better results than $\operatorname{Sim} 4 \mathrm{cc}$

We found 30,194 rice genes with at least one intron by BLAT, but the number was 30,177 according to the annotated information. Because the BLAT results were larger than the annotated results, the BLAT results must have predicted some new and different genes with introns. In the BLAT results, many short-length introns (less than $50 \mathrm{bp}$ ) were predicted, but in fact, these short-length introns were part of transcript sequences and were not real intron sequences. In contrast, Sim4cc detected 29,875 genes with introns, and all of these genes were contained in the annotation information. The predicted intron accuracy rate of Sim4cc was $100 \%$. On accuracy, Sim4cc was better than BLAT.

If Sim4cc is used, the user has to splice a whole genome file to many files: one gene, one file. The computing process of Sim4cc was more complex than that of BLAT, and each time, Sim4cc only calculated one cDNA sequence to one gene sequence; so, the executing efficiency and speed are not high. In comparison, BLAT was easier and faster than Sim4cc.

In conclusion, BLAT and Sim4cc can be used to predict introns, but each of them has its advantages and disadvantages. The comparative results are summarized in Table 5. Sim4cc was a cross-species spliced alignment program. In our study, Sim4cc was used to find introns by comparing cDNA sequences and gene sequences. The correct intron can be obtained by comparing one cDNA sequence with its own gene sequence. But, a lot of introns were lost by Sim4cc. In other words, Sim4cc was good at detecting the correct intron but not at predicting the whole number of introns in a genome. In contrast, BLAT can predict most of the introns nearly all of the total introns in a genome. But, there were some false-positive predictions of introns. However, the proportion of this error was very small. As a result, BLAT will be proposed to annotate plant genome introns.

\section{Intron length distribution of 10 plants}

According to Roy's method, many predicted introns in the plant genomes had in-frame stop codons, and the predicted introns in these genomes were equally as likely to be a multiple of $3 \mathrm{bp}(3 \mathrm{n})$ as to contain a plus one $(3 n+1)$ or two $(3 n+2)$ bp. Here was an example of three phases from an Arabidopsis thaliana gene, AT1G17600.1 (Fig. 2).

By analyzing genome sequence annotations, we got three-phase intron distributions for 10 plant species (Table 6). If the plant intron annotation is more accurate, the number of three phases should be similar (one-third each). For $80 \%(8 / 10)$ of species, there were similar numbers of the three phases. It should be noted that most of these plant species annotations were the best annotations to date, but new annotations will be continually released to correct errors and false-positive results.

\section{Two-species $3 n$ intron skew analysis}

For all of the 10 genomes (Table 6), there were very similar numbers of $3 n+1$ and $3 n+2$ introns, and the percentages of $3 n+1$ and $3 n+2$ introns were within $0.8 \%$. In contrast, the number of $3 n$ introns varied much more widely, from $29.1 \%$ to $47.7 \%$. In this study, two species' genome introns showed strongly skewed per- 
Intron $13 \mathrm{n}$

... CAA GCC ACT Ggt aag cet cgt ttt ctt gtt tac aca cat tta tca ctt tgt tta gca gca cac tgg aaa gtt gaa tta taa ttt tcc tgc tca att tca ata tta tta $\mathrm{gTG}$ TTG ATG AGG ...

Intron $23 n+2$

... TCA GAG ACg taa gca tct ata tca tct ttg atc tat tct ttt aaa ttt tca tgc atc ctg acc tga cga gtt tct ggc ttt gtg ttt ctt $t$ tg tct tct tat cat cag **G GAG GAG AAC ...

Intron $33 \mathrm{n}+1$

... AAA CAA GGA Ggt gaa tac ttg get ctt gat ccg tct cta cta tga ttg atg tag tta ccc ttt atc atc tcc ctt ctt tta tag *GC ACA TAC ACG ...

Fig. 2. An example of three phases of intron from an Arabidopsis gene, AT1G17600.1. Upper/lowercase sequence indicates exon/intron sequence. Asterisks indicate frameshifts introduced by non-3n introns; intronic in-frame stop codons are underlined. Intron 1 is a 99-bp intron (3n) with one in-frame stop codon. Intron 2 is a 100-bp intron (3n +2$)$, which has two in-frame stop codons and thus does not interrupt the open reading frame. Intron 3 is a 74-bp intron $(3 n+1)$ with three stop codons.

Table 6. Intron three-phase distributions of 10 plant species

\begin{tabular}{lrcccrrr}
\hline \multicolumn{1}{c}{ Species } & Intron No. & $3 n$ & $3 n+1$ & $3 n+2$ & Excess $3 n$ & $(3 n+1)-(3 n+2)$ \\
\hline Arabidopsis thaliana & 175,513 & 0.333 & 0.334 & 0.334 & 0.001 & 0.000 \\
Oryza sativa L. ssp. japonica & 251,812 & 0.353 & 0.322 & 0.324 & -0.030 & -0.002 \\
Oryza sativa L. ssp. indica & 127,029 & 0.329 & 0.335 & 0.335 & 0.006 & 0.000 \\
Zea mays & 266,772 & 0.331 & 0.335 & 0.334 & 0.003 & 0.001 \\
Sorghum bicolor & 115,610 & 0.336 & 0.334 & 0.331 & -0.004 & 0.003 \\
Cucumis sativus & 90,434 & 0.331 & 0.334 & 0.335 & 0.003 & 0.000 \\
Chlamydomonas reinhardtii & 104,660 & 0.355 & 0.323 & 0.322 & -0.033 & 0.001 \\
Ostreococcus lucimarinus & 2,369 & 0.477 & 0.258 & 0.265 & -0.215 & -0.007 \\
Ostreococcus tauri & 4,334 & 0.291 & 0.358 & 0.350 & 0.063 & 0.008 \\
Medicago truncatula & 152,466 & 0.331 & 0.336 & 0.333 & 0.004 & 0.002 \\
\hline
\end{tabular}

centages, in that the $3 \mathrm{n}$ intron percentage was much lower or higher than the expected value (one-third). Such a skew suggests systematic errors in the intron prediction.

The green alga Ostreococcus lucimarinus has one of the highest gene densities known in eukaryotes, with many introns [28]. There was a striking excess of predicted $3 \mathrm{n}$ introns $(47.7 \%$ of all predicted introns, 1,130$)$ compared to $3 n+1(25.8 \%, 611)$ and $3 n+2(26.5 \%$, 628) introns. In this case, many predicted $3 n$ introns were not true introns but instead exons.

The unicellular green alga Ostreococcus tauri is the world's smallest free-living eukaryote known to date [29]. These predicted introns showed a deficit of $3 n$ introns $(29.1 \%, 1,262)$, much lower than $3 n+1(35.8 \%, 1,553)$ and $3 n+2(35 \%, 1,519)$ introns. This result is very close to previous studies [18]. In this case, $3 n$ introns may be mistakenly regarded as coding sequences, whereas a $3 n+1$ or $3 n+2$ intron may be inferred from the disruption of the coding frame.

\section{Concluding remarks}

By comparing the advantages and disadvantages of BLAT and Sim4cc in intron prediction, we found that BLAT is faster and can predict more introns than Sim4cc. Through using intron length distribution to detect introns' annotations, it is a simple and fast method for detecting a variety of possible systematic biases in intron prediction or even for detecting problems with genome assemblies.

\section{Acknowledgments}

This study was funded by the Korea Science and Engineering Foundation, the National Natural Science Foundation of China (No. 30900780), the China Postdoctoral Science Foundation (No. 20090461260 \& No. 201104647), and the Postdoctoral Foundation of Shandong Agricultural University (No. 76267). 


\section{References}

1. Purdom E, Simpson KM, Robinson MD, Conboy JG, Lapuk AV, Speed TP. FIRMA: a method for detection of alternative splicing from exon array data. Bioinformatics 2008;24:1707-1714.

2. Zhou L, Pertea M, Delcher AL, Florea L. Sim4cc: a cross-species spliced alignment program. Nucleic Acids Res 2009;37:e80.

3. Harrington ED, Bork P. Sircah: a tool for the detection and visualization of alternative transcripts. Bioinformatics 2008;24:1959-1960.

4. Rambaldi D, Felice B, Praz V, Bucher P, Cittaro D, Guffanti A. Splicy: a web-based tool for the prediction of possible alternative splicing events from Affymetrix probeset data. BMC Bioinformatics 2007;8 Suppl 1:S17.

5. Mitchell RA, Castells-Brooke N, Taubert J, Verrier PJ, Leader DJ, Rawlings CJ. Wheat Estimated Transcript Server (WhETS): a tool to provide best estimate of hexaploid wheat transcript sequence. Nucleic Acids Res 2007;35:W148-W151.

6. Lazzarato F, Franceschinis G, Botta M, Cordero F, Calogero RA. RRE: a tool for the extraction of non-coding regions surrounding annotated genes from genomic datasets. Bioinformatics 2004;20:2848-2850.

7. Milanesi L, Rogozin IB. ESTMAP: a system for expressed sequence tags mapping on genomic sequences. IEEE Trans Nanobioscience 2003;2:75-78.

8. Wang K, Singh D, Zeng Z, Coleman SJ, Huang Y, Savich GL, et al. MapSplice: accurate mapping of RNA-seq reads for splice junction discovery. Nucleic Acids Res 2010;38:e178.

9. Dimon MT, Sorber K, DeRisi JL. HMMSplicer: a tool for efficient and sensitive discovery of known and novel splice junctions in RNA-Seq data. PLoS One 2010;5: e13875

10. Foissac S, Bardou $P$, Moisan $A$, Cros MJ, Schiex $T$. EUGENE'HOM: A generic similarity-based gene finder using multiple homologous sequences. Nucleic Acids Res 2003;31:3742-3745.

11. Kent WJ. BLAT: the BLAST-like alignment tool, Genome Res 2002;12:656-664.

12. Lee C, Atanelov L, Modrek B, Xing Y. ASAP: the Alternative Splicing Annotation Project. Nucleic Acids Res 2003;31:101-105

13. Odenwald WF, Rasband W, Kuzin A, Brody T. EVOPRINTER, a multigenomic comparative tool for rapid identification of functionally important DNA. Proc Nat/ Acad Sci U S A 2005;102:14700-14705.

14. Castrignanò T, De Meo PD, Grillo G, Liuni S, Mignone F, Talamo IG, et al. GenoMiner: a tool for genome-wide search of coding and non-coding conserved sequence tags. Bioinformatics 2006;22:497-499.

15. Tamaki S, Arakawa K, Kono N, Tomita M. Restauro-G: a rapid genome re-annotation system for comparative genomics. Genomics Proteomics Bioinformatics 2007;5: 53-58.

16. Kent WJ, Zahler AM. The intronerator: exploring introns and alternative splicing in Caenorhabditis elegans. $\mathrm{Nu}$ cleic Acids Res 2000;28:91-93.

17. Irimia M, Roy SW. Spliceosomal introns as tools for genomic and evolutionary analysis. Nucleic Acids Res 2008;36:1703-1712.

18. Roy SW, Penny D. Intron length distributions and gene prediction. Nucleic Acids Res 2007;35:4737-4742.

19. Swarbreck D, Wilks C, Lamesch P, Berardini TZ, GarciaHernandez $\mathrm{M}$, Foerster $\mathrm{H}$, et al. The Arabidopsis Information Resource (TAIR): gene structure and function annotation. Nucleic Acids Res 2008;36:D1009-D1014.

20. Goff SA, Ricke D, Lan TH, Presting G, Wang R, Dunn $\mathrm{M}$, et al. A draft sequence of the rice genome (Oryza sativa L. ssp. japonica). Science 2002;296:92-100.

21. Yu J, Hu S, Wang J, Wong GK, Li S, Liu B, et al. A draft sequence of the rice genome (Oryza sativa $\mathrm{L}$. ssp. indica). Science 2002;296:79-92.

22. Schnable PS, Ware D, Fulton RS, Stein JC, Wei F, Pasternak S, et al. The B73 maize genome: complexity, diversity, and dynamics. Science 2009;326:1112-1115.

23. Paterson AH, Bowers JE, Bruggmann R, Dubchak I, Grimwood J, Gundlach $\mathrm{H}$, et al. The Sorghum bicolor genome and the diversification of grasses. Nature 2009; 457:551-556.

24. Han YH, Zhang ZH, Liu JH, Lu JY, Huang SW, Jin WW. Distribution of the tandem repeat sequences and karyotyping in cucumber (Cucumis sativus L.) by fluorescence in situ hybridization. Cytogenet Genome Res 2008;122:80-88

25. Merchant SS, Prochnik SE, Vallon O, Harris EH, Karpowicz SJ, Witman GB, et al. The Chlamydomonas genome reveals the evolution of key animal and plant functions. Science 2007;318:245-250.

26. Palenik B, Grimwood J, Aerts A, Rouzé P, Salamov A, Putnam $\mathrm{N}$, et al. The tiny eukaryote Ostreococcus provides genomic insights into the paradox of plankton speciation. Proc Natl Acad Sci U S A 2007;104:77057710.

27. Young ND, Cannon SB, Sato S, Kim D, Cook DR, Town CD, et al. Sequencing the genespaces of Medicago truncatula and Lotus japonicus. Plant Physiol 2005;137:1174-1181.

28. Lanier W, Moustafa A, Bhattacharya D, Comeron JM. EST analysis of Ostreococcus lucimarinus, the most compact eukaryotic genome, shows an excess of introns in highly expressed genes. PLOS One 2008;3:e2171.

29. Derelle E, Ferraz C, Rombauts S, Rouzé P, Worden AZ, Robbens $\mathrm{S}$, et al. Genome analysis of the smallest free-living eukaryote Ostreococcus tauri unveils many unique features. Proc Natl Acad Sci U S A 2006;103: 11647-11652. 